\title{
Análisis del impacto del crédito para iniciativas productivas entregados a los beneficiarios del bono de desarrollo humano. Estudio de caso
}

\author{
Barrerno Benavides, Luis; López Paredes, Héctor; Cabrera Maya, Luis \\ Análisis del impacto del crédito para iniciativas productivas entregados a los beneficiarios del bono de desarrollo \\ humano. Estudio de caso \\ Revista Economía y Política, núm. 31, 2020 \\ Universidad de Cuenca, Ecuador \\ Disponible en: http://www.redalyc.org/articulo.oa?id=571162102003 \\ DOI: https://doi.org/10.25097/rep.n31.2020.01 \\ La Universidad de Cuenca en Ecuador, conserva los derechos patrimoniales (copyright) de las obras publicadas, \\ y favorece y permite la reutilización de las mismas bajo la licencia Creative Commons Atribución-NoComercial- \\ Compartirlgual 4.0 Internacional (CC BY-NC-SA 4.0), por lo cual se pueden copiar, usar, difundir, transmitir y \\ exponer públicamente, siempre que: a. Se cite la autoría y fuente original de su publicación (revista, editorial, \\ URL y DOI de la obra). b. No se usen para fines comerciales u onerosos. c. Se mencione la existencia y \\ especificaciones de esta licencia de uso. \\ Esta obra está bajo una Licencia Creative Commons Atribución-NoComercial-Compartirlgual 4.0 Internacional.
}


Artículos

\title{
Análisis del impacto del crédito para iniciativas productivas entregados a los beneficiarios del bono de desarrollo humano. Estudio de caso
}

\author{
Analysis of the impact of credit for productive initiatives delivered to the beneficiaries of the human development \\ bond. Case study \\ Luis Barrerno Benavides \\ Universidad UTE, Ecuador \\ lbarreno@ute.edu.ec \\ DOI: https://doi.org/10.25097/rep.n31.2020.01 \\ Redalyc: http://www.redalyc.org/articulo.oa? \\ id $=571162102003$
}

iD http://orcid.org/0000-0001-5372-2355

Héctor López Paredes

Universidad UTE, Ecuador

hector.lopez@ute.edu.ec

iD http://orcid.org/0000-0003-0456-5271

Luis Cabrera Maya

Universidad UTE, Ecuador

luisg.cabrera@ute.edu.ec

iD http://orcid.org/0000-0002-2120-1448

Recepción: 14 Junio 2019

Aprobación: 06 Diciembre 2019

\section{RESUMEN:}

El propósito del artículo es analizar las percepciones de las personas que han accedido a créditos productivos siendo beneficiarias del Bono de Desarrollo Humano (en adelante se lo citará como BDH), considerando como caso de estudio la zona rural de la Provincia de Pichincha - Ecuador, tomando una muestra representativa para el análisis de las opiniones de este grupo poblacional en relación a los impactos socio económicos del crédito en la mejora de sus ingresos y condiciones de vida, entendiendo paralelamente, la complejidad de trámites administrativos, tiempo de entrega de recursos, asesoría técnica y seguimiento brindado, así como, las recomendaciones manifestadas por los usuarios del crédito.

La investigación contribuye con mayores elementos de juicio al gobierno e instituciones relacionadas a la concesión de créditos para iniciativas productivas en el Ecuador y países de similar situación, permitiendo definir acciones tendientes a la continuidad y mejora de los mismos en función a la cualificación brindada a los beneficiarios.

Palabras Clave: Bienestar social, crédito, desarrollo humano, emprendimientos productivos, protección social.

\section{Abstract:}

The purpose of the article is to analyze the perceptions of people who have accessed productive credits being beneficiaries of the Human Development Bond (BDH), considering as a case study the rural area of the Province of Pichincha - Ecuador, taking a representative sample for the analysis of the opinions of this population group in relation to the socio-economic impacts of credit in improving their income and living conditions, understanding in parallel, the complexity of administrative procedures, time of delivery of resources, technical advice and monitoring provided, as well as, the recommendations expressed by credit users.

The investigation contributes with greater elements of judgment to the government and institutions related to the granting of credits for productive initiatives in Ecuador and countries of similar situation, allowing to define actions aimed at the continuity and improvement of the same depending on the qualification provided to the beneficiaries.

KEYWORDS: Credit, human development, productive undertakings, social protection, social welfare. 


\section{INTRODUCCIÓN}

Las políticas de desarrollo humano constituyen una posibilidad de mejora para los segmentos de la población que se encuentran en condiciones de pobreza y pobreza extrema, definiendo los mecanismos que les permiten acceder a créditos y bonos otorgados por el gobierno con la finalidad de cambiar su situación, en donde, siguiendo lo planteado por Villatoro y Silva (2005) es el Estado el responsable de garantizar a la población los medios y recursos que faciliten su mejora, conjugando el desarrollo orientado a los derechos con el diseño de instituciones y políticas que permitan producir y asignar los recursos necesarios para darles un carácter efectivo.

En el caso de Ecuador, se carece de medios que permitan evidenciar el efecto de estas políticas en la sociedad, desconociendo si han cumplido con su propósito, planteando como hipótesis de investigación: La ejecución y la operación de los pequeños emprendimientos productivos financiados con créditos otorgados a los beneficiarios del BDH, han permitido mejorar su percepción de mejora en su nivel de vida; caso contrario, estas prácticas para Stefanoni (2012) serán únicamente un discurso de izquierda sobre eliminación de pobreza pues, esta ayuda económica no debe desincentivar la participación de la fuerza de trabajo (Calderón, 2012), siendo para Gasparini, Cicowiez, y Sosa (2014) la pobreza y la desigualdad problemas sociales centrales en América Latina que deben ser estudiados para comprender la desigualdad social en términos absolutos y relativos.

Un indicador que puede contribuir a demostrar la hipótesis planteada, además de la percepción de los propios beneficiarios del crédito y que ayuda al entendimiento de los efectos del BDH en la sociedad: es el índice de pobreza, que según el Instituto Ecuatoriano de Estadística y Censo (INEC, 2018) pasó de 36.7\% en el año 2007 al 22,9\% a finales del año 2016, detalle que se explica más adelante. Con los datos señalados se puede colegir que la distribución en el país mejoró y uno de los instrumentos, no el único, fue el BDH y concomitantemente se entendería el crédito productivo derivado de él.

Según Rosero y Ramos (2016) a partir del llamado gobierno de la "Revolución Ciudadana" se busca implementar políticas públicas que acorten las brechas de pobreza e impulsen la inclusión económica y social en el país como parte de la planificación para el "Buen Vivir"; constituyéndose este bono como una transferencia que incrementa de manera directa el ingreso de la familia para reducir la vulnerabilidad del hogar. El estudio de Cabezas (2018) evidencia un crecimiento pro pobre encontrado en la economía ecuatoriana para el período 2006-2014, como efecto de las funciones de distribución para los ingresos, así como para los gastos reales, cambiando favorablemente para los estratos sociales más pobres. Sin embargo, Guamán, Lara, Alvarado, y Ponce (2019) en relación al BDH manifiestan que la transferencia monetaria condicionada no necesariamente está cumpliendo los objetivos del programa, en consecuencia, las políticas sociales deberían garantizar que este tipo de programas no sean un instrumento de dependencia que limiten las capacidades individuales, debido a que esto impide la reducción de la pobreza y la desigualdad.

Con lo expresado, este estudio es fundamental para entender el efecto de estas políticas en los grupos sociales a los cuales se dirige, analizando el impacto del crédito para iniciativas productivas entregadas a los beneficiarios del BDH desde la percepción de mejora sobre sus condiciones de vida, manteniendo sus principales implicaciones teóricas en el respaldo de las políticas de protección y desarrollo social, identificando como principal responsable de este cambio las políticas de gobierno tendientes a estimular el desarrollo social a través de acciones que faciliten el acceso de personas en condición de pobreza y pobreza extrema a los medios necesarios para emprender, producir e incorporarse a la población económicamente activa; definiendo como implicaciones prácticas: (1) la necesidad de definir los medios necesarios de seguimiento y control del dinero entregado a este grupo social, a fin de tener información más clara sobre su evolución, (2) el mantenimiento de este tipo de créditos que estimulan el desarrollo productivo en segmentos sociales vulnerables y en zonas geográficas de difícil acceso y, (3) el desarrollo de programas de formación 
para los beneficiarios del BDH y de los créditos productivos, a fin de que los recursos entregados tengan un respaldo técnico de aplicación, así como, el asesoramiento necesario para su mantenimiento y mejora.

\section{REVISIÓN DE LITERATURA}

\section{Protección Social y el Bono de Desarrollo Humano}

El Bono de Desarrollo Humano (BDH) nace como parte de una política de protección social por parte del Estado, expresada esta última, como una declaratoria de disminución de la pobreza y desigualdad social (Villatoro y Silva, 2005), en la que se manifiesta que en la región se presentan procesos de movilidad social ascendente muy importantes relacionados con los niveles de crecimiento económico sostenido, al igual que con cambios en los patrones de distribución primaria y de redistribución de la riqueza, a pesar de lo cual los desafíos de erradicación de la pobreza y desigualdad social se mantienen presentes, siendo necesaria la construcción e implementación de sistemas inclusivos de protección social.

Armas (2005) indica que el BDH constituyó un programa de transferencia de recursos económicos, condicionada a la inversión en educación y salud, dirigido a los hogares más pobres, en los que destaca la transferencia a madres, cabeza de hogar de dichos segmentos; sin embargo, en lo relativo a educación o a elevar el nivel cognitivo de los niños/as de esos hogares, Ponce, Enríquez, Molyneux, y Thomson (2013) señalan que el BDH no ha generado ningún impacto a ese nivel, o al menos no existe evidencia empírica que demuestre lo contrario, manifestando que se requiere intervenciones adicionales y alternativas, siendo corroborado en el estudio de Calvas (2010) que demuestra que el BDH no presentó ninguna evidencia estadísticamente significativa de mejora en la inserción educativa.

Stefanoni (2012) señala que las políticas de gobierno que colocan al ser humano por sobre el capital impulsan la generación de beneficios sociales como bonos, créditos, mejoras en los servicios de salud y educación, entre otros, mismos que se derivan de problemas microeconómicos en los que se requiere generar empleo productivo y de calidad. Con lo expresado se puede entender a la protección social como un eje de acción de los gobiernos con enfoque de izquierda que buscan erradicar la pobreza y disminuir las brechas sociales. En este enfoque, los subsidios, bonos u otro tipo de ayudas se han constituido, para los distintos gobiernos, en instrumentos para apoyar a grupos sociales más vulnerables.

La crisis socio económica del Ecuador a finales de los 90's, impulsó a generar el BDH, el cual surge en 1998 con el Decreto Ejecutivo 129 como una compensación para la población pobre por la eliminación del subsidio al gas, la electricidad y el combustible, mismo que es entregada por bancos privados y el Banco Nacional de Fomento actualmente conocido como BAN Ecuador, siendo importante precisar que el BDH no surge como una política de lucha contra la pobreza, sino como una compensación. En el 2003 con Decreto Ejecutivo 347 el BDH pasa a ser un programa que busca entregar un subsidio monetario de protección social administrado por el Ministerio de Bienestar Social, manteniendo hasta la actualidad varias reformas que han permitido brindar más beneficios a quienes lo perciben, en donde, según datos del SIISE (2013): el representante del núcleo familiar (madres) reciben $\$ 50$ dólares mensuales sujetos a corresponsabilidades en salud y educación, pensión asistencial para adultos mayores quienes reciben $\$ 50$ dólares mensuales, pensión asistencial para personas con discapacidad quienes reciben $\$ 50$ dólares mensuales, acreditados con $40 \%$ o más de discapacidad de acuerdo al carnet del Consejo Nacional de Discapacidades (que en adelante se lo citará como CONADIS).

Conforme lo expresado por el Ministerio de Inclusión Económica y Social (2016) (en adelante se lo citará como MIES), a partir del llamado gobierno de la "Revolución Ciudadana" [1] se busca implementar políticas públicas que acorten las brechas de pobreza e impulsen la inclusión económica y social en el país como parte 
de la planificación para el "Buen Vivir" [2] ; siendo este bono una transferencia que incrementa de manera directa el ingreso de la familia para reducir la vulnerabilidad del hogar.

Un indicador que puede contribuir al BDH es el índice de pobreza que según el Instituto Ecuatoriano de Estadística y Censo (INEC, 2018) pasó de 36.7\% en el año 2007 al 22,9\% a finales del año 2016; más, para evidenciar la desigualdad de la distribución del ingreso en el país se ha usado el coeficiente de Gini ${ }^{[3]}$, que para 1999 (año del feriado bancario) fue de 0.58; en 2007 (inicio del mandato de Rafael Correa) alcanzó el 0.54, terminando en el año 2017 en 0.46 (fin del período presidencial de Rafael Correa). Por los datos señalados se puede colegir que la distribución en el país mejoró y uno de los instrumentos, no el único, fue el BDH y concomitantemente el crédito productivo derivado de él; sin embargo, Guamán, et al. (2019) señalan que las políticas deben ir encaminadas a reforzar y asegurar una adecuada distribución de esta ayuda económica y verificar el correcto cumplimiento de los requisitos básicos de elegibilidad.

\section{Desarrollo Humano}

Calvas (2010) hace referencia al desarrollo humano como el proceso de ampliar las opciones de las personas, siendo su principal objetivo permitir que estas disfruten de vidas prolongadas, saludables y creativas, así como, crear un entorno en el que las personas puedan hacer plenamente realidad sus posibilidades y vivir en forma productiva y creadora de acuerdo con sus intereses, por lo que para que exista desarrollo y este sea sostenible según Artaraz (2002) se deben considerar 3 dimensiones: la primera es la dimensión económica en la que se contempla los recursos que se emplean para la producción; la segunda se refiere a la dimensión social en la que se encuentra implícito el principio de equidad y que abarca la gestión de los conflictos presentes en la sociedad en cuanto a la organización y desarrollo de los medios de producción, así como el propio relacionamiento entre individuos; y la tercera dimensión que contempla lo ecológico, es decir, un concepto de producción cíclica que imita a la naturaleza, entendiendo que no se generan desperdicios ni afecta al entorno y la naturaleza, en la que se incorpora el Índice de Desarrollo Humano generando por las Naciones Unidas en 1990, que mide el progreso de un país a partir de la esperanza de vida, el nivel educacional y el ingreso per cápita.

La Comisión Económica para América Latina (CEPAL 2006) señala que el desarrollo humano demanda de políticas de protección social que se centren en derechos humanos, sociales y ciudadanos en los cuales se promueva una dinámica contributiva entre el mercado laboral y la política fiscal, en donde, su resultado se exprese en planes y programas de beneficio social que favorezca el acceso a salud, educación, vivienda, trabajo, jubilación, entre otros; que, para Paladines (2016) deben apegarse al paradigma de maximización del bienestar social que distingue entre principios "eco-nómicos" y éticos para la existencia de un equilibrio general perfectamente competitivo y descentralizado.

\section{Crédito de Desarrollo Humano}

De acuerdo a Coba y Díaz (2014) el BDH busca que las familias y comunidades se agrupen para desarrollar actividades productivas, evolucionando de un programa de transferencia monetaria a un incentivo de producción y auto trabajo, dando origen a negocios en actividades agrícolas, pecuarias, artesanales, comerciales, así como de ayuda social, muestra de ello es el estudio realizado por Medina (2016) en el que se evidencia la incidencia favorable del $\mathrm{BDH}$ en la potencialización de las capacidades productivas de las mujeres de la Isla Trinitaria en Guayaquil - Ecuador, al igual que lo señalado por el (Ministerio de Industrias y Productividad, 2017) en su sitio web respecto a la necesidad de impulsar el crédito para emprendimientos productivos de tipo individual y asociativo a partir de los recursos provenientes de créditos del $\mathrm{BDH}$, en el que se cuenta con proyectos como el Fondepyme ${ }^{[4]}$ y Exportapyme ${ }^{[5]}$ orientados a promover y apoyar 
actividades productivas, así como los créditos que se impulsan a través de BanEcuador y el programa Progresar que impulsa la Corporación Financiera Nacional (CFN).

Acosta y Falconí (2005) plantean, tomando como referencia la crisis que experimentó Ecuador a finales de los 90, que cualquiera de estos cambios, más allá de un concepto político neoliberal, progresista, socialista o cualquier otro, deben responder a un modelo de país construido socialmente, en donde el Estado recupere el carácter público y lo desencadene a los intereses corporativos privados, impulsando mejores condiciones de acceso a empleo, salud y educación, al igual que garantice la distribución equitativa de la riqueza y disminuya las brechas de pobreza. Entonces, el Estado de bienestar contempla dos funciones básicas la redistribución y reproducción en las cuales, de acuerdo a Acosta y Falconí (2005) se debe intervenir en la lógica del mercado para lograr:

- Des mercantilizar, redistribuir y articular dinámicas de empleo y movilidad social.

- Generar capital humano o fuerza de trabajo; tarea que es realizada básicamente en los hogares y el Estado complementa.

- Mantener un nivel mínimo de calidad de vida de las personas, para crear capacidad de respuesta independiente de los ciclos económicos; factor relacionado con el nivel de gasto social y la atención a las personas que se considera más pobres o vulnerables ante recesiones.

Estos aspectos obligaron en el país a pensar en una planificación que incorpore principios de desarrollo humano, que fueron incluidos en el Plan Nacional del Buen Vivir (PNB), desde su primera versión en el 2007, así el Consejo Nacional de Planificación (2017) expresa en el PNB 2017 - 2021 la necesidad de no abstraer el desarrollo humano de la naturaleza ya que en ella se desenvuelve la vida, siendo su sostenibilidad una responsabilidad ética con la generaciones futuras. Vos (2000) manifestó la necesidad del Ecuador de incorporar políticas que estabilicen su economía y promuevan un crecimiento basado en el desarrollo social, es decir, que se exprese en una mejor educación, seguridad, salud, trabajo y demás aspectos relacionados al estado de bienestar humano. Con lo cual se hace importante considerar las funciones de la política social que promueven un Estado de bienestar, en la que según Acosta y Falconí (2005) son:

- Mantener niveles de vida adecuados en la población; entendida por la reducción de la pobreza, la seguridad y la suavización del ingreso.

- Redistribuir, con el fin de reducir la desigualdad bajo fuertes principios de equidad y justicia.

- Reproducción y movilidad social, relacionada a la inclusión, participación, solidaridad social y la libertad que promueven derechos y obligaciones de convivencia social.

El crédito para emprendimientos productivos como parte del bono de desarrollo humano tiene su origen en el BDH creado en 1998 mediante decreto ejecutivo 129, como una especie de compensación para la población pobre a la eliminación de algunos subsidios como el gas, la energía eléctrica, y los combustibles, luego en el 2003, mediante Decreto ejecutivo 347, pasa a ser un programa para entregar un subsidio monetario de protección social administrado por el MIES. A partir del año 2008, según el Sistema Integrado de Indicadores Sociales del Ecuador (SIISE, 2010), se estable el actual Crédito de Desarrollo Humano $(\mathrm{CDH})$, administrado por el Programa de Protección social (PPS), teniendo como destinatarios a madres, adultos mayores, personas con discapacidad, como una línea de crédito dentro del Programa Crédito Productivo Solidario.

La finalidad de este crédito es financiar actividades de producción, comercio o servicio y de esta forma potenciar el desarrollo de habilidades y destrezas productivas en los beneficiarios del crédito, pudiendo invertir en pequeños negocios que generen ganancias en emprendimientos de su propiedad y administración propia, que generalmente no tienen empleados siendo más bien unipersonales, todo esto para motivar e incentivar la producción por parte de la población de escasos recursos económicos y de esta forma mejorar la generación de ingresos que tenga un efecto favorable en la mejora de su nivel de vida, respaldando lo señalando por Cabezas (2018) al referir que las políticas públicas que permitieron incrementar las dotaciones y el retorno del capital humano para las personas con menor nivel educativo, permitió que los individuos 
ubicados hasta el percentil 30 tuvieran tasas de crecimiento en ingreso real superiores al $9 \%$ anual promedio, indicador que es significativamente diferente al PIB per cápita real, llevando a una convergencia entre clases sociales.

\section{Emprendimientos productivos}

Si bien el emprendimiento tiene un término polisémico como el conjunto de actitudes y conductas que dan lugar a un determinado perfil personal orientado hacia la autoconfianza, la creatividad, la capacidad de innovación, el sentido de responsabilidad y el manejo del riesgo (Salinas y Osorio, 2012); en esta definición prácticamente el ser humano es el centro y el todo del emprendimiento mediante una serie de virtudes propias del buen emprendedor, recayendo sobre él toda la responsabilidad del éxito o fracaso, por tanto, para entender los créditos productivos destinados a los beneficiarios del $\mathrm{BDH}$ en un proyecto de gobierno que busca fomentar el emprendimiento, se debe considerar las seis características recomendadas por Patanakul, Kwak, Zwikael, y Liu (2016):

1) Perseguir beneficios de objetivos no financieros;

2) Tener una larga vida útil del producto;

3) Tratar con múltiples partes interesadas;

4) Ser un megaproyecto grande y complejo;

5) Ser susceptible al entorno y la dinámica política; y

6) Seguir un proceso de gestión de proyectos obligatorio.

En el caso de Ecuador, para la selección de los sectores censales más pobres se utiliza la información cartográfica del Instituto Nacional de Estadísticas y Censos (INEC) y el Índice de Necesidades Básicas Insatisfechas desarrollado por la Comunidad Andina de Naciones, identificando los sectores censales donde la incidencia de la pobreza supera al 50\% de los hogares, en donde, el último levantamiento de información socioeconómica en 2013 indica 24.482 sectores en los cuales debe enfocarse el BDH (Martínez, Borja, Medellín, y Cueva, 2017). Esta sectorización debe promover programas de transferencias monetarias condicionadas que impulsen emprendimientos productivos sostenibles y sustentables por si solos, para eliminar la dependencia de programas de protección social y subsidios, teniendo en cuenta que estudios como el realizado por Barreno, et al. (2018) demuestran que la mayoría de emprendimientos productivos provienen del sector rural o tienen su origen en el mismo a través de la generación de una asociación de economía social y solidaria.

\section{Materiales Y MÉtodos}

El diseño metodológico fue de carácter mixto, el cual según Hernández y Mendoza (2018) involucra la recolección y análisis de datos cualitativos y cuantitativos, así como, su integración y discusión conjunta para un mejor entendimiento de las variables de estudio, en donde, la revisión cualitativa de datos secundarios y del marco teórico fue de tipo exploratoria y documental, para comprender la evolución del concepto de desarrollo humano, su aplicación e incorporación en programas políticos de desarrollo social, y su efecto en las condiciones de vida de los beneficiarios de estas prácticas, permitiendo conocer la realidad de la provincia de Pichincha como caso de estudio, alrededor del impacto del crédito para iniciativas productivas entregados a los beneficiarios del BDH, en el período de gobierno del Econ. Rafael Correo. Esta información fue contrastada con datos primarios obtenidos del cuestionario desarrollado por los autores en base a las variables analizadas en la revisión de la literatura, en donde, siguiendo los lineamientos establecidos por Hernández y Mendoza (2018) la información resultante de una rigurosa revisión documental fue validada a través de técnicas estadísticas complejas, que permitieron a través del procesamiento y análisis 
de datos recabados medir las variables de estudio, establecer su causalidad, determinar los coeficientes estadísticos necesarios, como el Alfa de Crombach, que permitió obtener el análisis de fiabilidad del cuestionario y las combinaciones de las variables más importantes consultadas, como la continuación actual del emprendimiento, la percepción del beneficiario del BDH sobre la mejora en su calidad de vida, obteniéndose un nivel de fiabilidad del 71.7\%, lo que según González y Pazmino (2015) los valores entre 0.70 y 0.90 indica una buena consistencia interna para una escala unidimensional, como una medida que cuantifica la correlación existente entre los ítems que la componen (ver tabla 1 y tabla 2).

TABLA 1.

Resumen de procesamiento de casos

\begin{tabular}{llll} 
& \multicolumn{1}{c}{$\mathrm{N}$} & $\%$ \\
\hline Casos & Válido & 414 & 100,0 \\
\cline { 2 - 4 } & Excluido $^{2}$ & 0 & 0 \\
\cline { 2 - 4 } & Total & 414 & 100,0 \\
\hline \multirow{2}{*}{ a. La eliminación por lista se basa en }
\end{tabular}

todas las variables del procedimiento.

Elaborado por los autores.

TABLA 2.

Estadísticas de fiabilidad

\begin{tabular}{l|l}
$\begin{array}{l}\text { Alfa de } \\
\text { Cronbach }\end{array}$ & $\begin{array}{l}\text { N de } \\
\text { elementos }\end{array}$ \\
\hline 717 & 2 \\
\hline
\end{tabular}

Elaborado por los autores.

Para la recolección de la información de primera mano de los sujetos investigados se utilizó información primaria a través de la investigación de campo utilizando el sistema de muestreo, tomando una muestra representativa de tipo probabilística, por cuanto, toda la población que recibió el crédito tiene la misma opción de ser escogidos, pudiendo generalizar los resultados o hallazgos encontrados; luego se determinó la unidad de muestreo o a quién se aplicará la encuesta, en este caso el segmento de la población que recibió el crédito para emprendimientos productivos ubicados en el sector rural de la provincia de Pichincha, el instrumento utilizado fue la encuesta, lo que permitió demostrar la pregunta central de investigación propuesta; tomando en cuenta en el universo de estudio a las personas que constan en la base de datos del (MIES), que siendo parte del programa de $\mathrm{BDH}$, recibieron un crédito para proyectos de emprendimientos productivos; el ámbito geográfico fue la Provincia de Pichincha en todos sus cantones y dentro de estos en el área rural, los cantones en los que se realizó la investigación fueron: Puerto Quito, Pedro Vicente Maldonado, San Miguel de los Bancos, Pedro Moncayo, Cayambe, Mejía y Quito; base de datos que fue actualizada al año 2016.

Para el análisis de los resultados de la investigación de campo, se utilizó el estudio de tipo correlacional, que permitió conocer la relación entre las variables que intervinieron en la investigación de campo a través de la aplicación del cuestionario, en donde, para determinar el tamaño óptimo de la muestra se aplicó el criterio de muestra finita, para lo cual se trabajó con una $(Z)$ o grados de confianza del $95 \%$ un nivel de error aceptable de un 5\%, obteniendo un total de 385 encuestas, sin embargo, para mayor fiabilidad se trabajó con un una población mayor correspondiente a 414 encuestas, que fueron procesadas con el Software estadístico SPSS, el cual permitió medir en primera instancia la confiabilidad de las encuestas a través del indicador Alfa de 
Combrach para las variables dependientes y luego a través de cruce de variables obtener los resultados que permitieron obtener las conclusiones y responder a la pregunta principal de la investigación.

Los resultados obtenidos permitieron el cumplimiento del objetivo de la investigación, el cual se logró mediante la información primaria obtenida con la técnica de investigación de campo a través de la aplicación de encuestas, la misma que en base a la pregunta principal de la investigación ęa partir de la operación del emprendimiento, en términos generales, qué pasó con su nivel de vida?, permitió determinar el impacto de este programa social implementado por el Gobierno del Presidente Rafael Correa.

Consideramos que la presente investigación pueda ser la pauta para nuevos estudios, que aborden con mayor profundidad el componente financiero, ventas, estructura de los emprendimientos u otra información empírica, y de esta manera corroborar o debatir los resultados o conclusiones alcanzados en esta investigación. Adicionalmente, nuevas incursiones académicas pueden priorizar factores como el componente etario, género o tipo de emprendimiento, entre otras.

\section{Resultados y Discusión}

Los resultados que se presentan a continuación, están en función de las respuestas a las preguntas generadas en la encuesta aplicada a los beneficiarios del BDH que han recibido créditos para emprendimientos productivos, los candidatos fueron extraídos de la base de datos entregada por el MIES en el 2016, siendo necesario recalcar que la presente investigación se basa en un estudio de caso y que corresponde a la zona rural de la provincia de Pichincha, por lo que cualquier extrapolación que se pretenda realizar a partir del estudio debe tomar en consideración esta condición.

Las formas utilizadas para la obtención del crédito fueron diversas, sin embargo, el mayor porcentaje 44.4\% presentó directamente, a la entidad proponente, los requisitos solicitados, el 29.2\% acudió directamente al MIESS para informarse, el 11.8\% lo hizo mediante una llamada telefónica y el 14,6\% lo realizó mediante otras formas; esto muestra que la entidad encargada de otorgar los préstamos les dio facilidades para la obtención del crédito, situación que fue favorable para el cumplimiento del objetivo del programa.

TABLA 3.

¿Cómo califica el grado de dificultad que tuvo durante el trámite realizado?

\begin{tabular}{llllll}
\hline & & Frecuencia & Porcentaje & $\begin{array}{l}\text { Porcentaje } \\
\text { válido }\end{array}$ & $\begin{array}{l}\text { Porcentaje } \\
\text { acumulado }\end{array}$ \\
\hline \multirow{4}{*}{ Válido } & $\begin{array}{l}\text { Muy } \\
\text { difícil }\end{array}$ & 18 & 4,3 & 4,3 & 4,3 \\
\cline { 2 - 6 } & Difícil & 118 & 28,5 & 28,5 & 32,9 \\
\cline { 2 - 6 } & Fácil & 278 & 67,1 & 67,1 & 100,0 \\
\cline { 2 - 6 } & Total & 414 & 100,0 & 100,0 & \\
\hline
\end{tabular}

Elaborado por los autores.

Como se puede observar en la Tabla 3, para el 67.1\% de los consultados resultó fácil acceder al crédito ofertado por la entidad oficial del gobierno, para un $28.5 \%$ resultó difícil y solo para un $4.3 \%$ resultó muy difícil, la razón que tuvieron la mayoría de las personas que respondieron esta última opción es por la dificultad de viajar de la zona rural a las oficinas del MIES en la ciudad de Quito para realizar los trámites. Estos datos muestran que el proceso de acceso al crédito no es complicado y que según datos del ONU (2015) y PNUD (2016) las políticas nacionales del Estado, así como, internacionales se orientan al desarrollo de los sectores marginales de los países en vías de desarrollo.

En relación al tiempo de demora en la entrega del crédito un $23,7 \%$ de los encuestados señalan que lo obtuvieron en menos de 15 días, el 10,1\% en quince días, el 24,2\% de beneficiarios lo obtuvieron en un mes, 
el 13.3\% lo obtuvieron en dos meses; es decir, más del 70\% de los beneficiarios del crédito lo obtuvieron en un corto plazo, que no supera los dos meses, lo cual indica cierto nivel de agilidad en la entrega del crédito, por lo que el tiempo de entrega de los desembolsos no representaría una dificultad, sin embargo, se debe considerar que para Calvas, (2010), Ángel (2012) y Rivera (2014) existen aspectos que deben ser analizados y controlados por el Estado para evitar que este tipo de políticas se conviertan en estrategias de aceptación popular, definiendo los mecanismos necesarios para su seguimiento y control.

De las 414 personas beneficiarias del crédito consultadas, se identificaron 89 distintos tipos de emprendimientos, en razón a que la investigación se realizó en zona rural, casi todos corresponden a actividades de tipo agropecuario, y dentro de estas, el mayor porcentaje corresponde al pecuario, en donde, predominan los emprendimientos sobre la crianza y venta de animales con el $34.1 \%$. Dentro del $22.7 \%$ de emprendimientos se encuentran una gama muy diversa, la mayoría de naturaleza comercial correspondientes a pequeños negocios, de compra y venta de productos, especialmente agropecuarios; con el 15.2\% están los emprendimientos de crianza de animales menores como son cuyes, conejos y aves de corral, en cuarto lugar, casi con el mismo porcentaje, está el comercio de animales combinados mayores y menores del cual el $10.4 \%$ corresponde a los emprendimiento de tipo agrícola, entre los que se destacan el cultivo de una serie de productos como hortalizas, legumbres, maíz, tubérculos, frutales, plantas ornamentales, entre otras, que representan actividades de poco fomento y que pueden caer en precarización salarial según lo expresado por Barreno, et al. (2018).

La Tabla 4 muestra si beneficiario del crédito recibió una capacitación previa:

TABLA 4.

Tabla cruzada. Cuando recibió el dinero ¿obtuvo alguna capacitación referente al emprendimiento productivo que iba a realizar?* A partir de la operación del emprendimiento en términos generales ¿qué pasó con su nivel de vida?

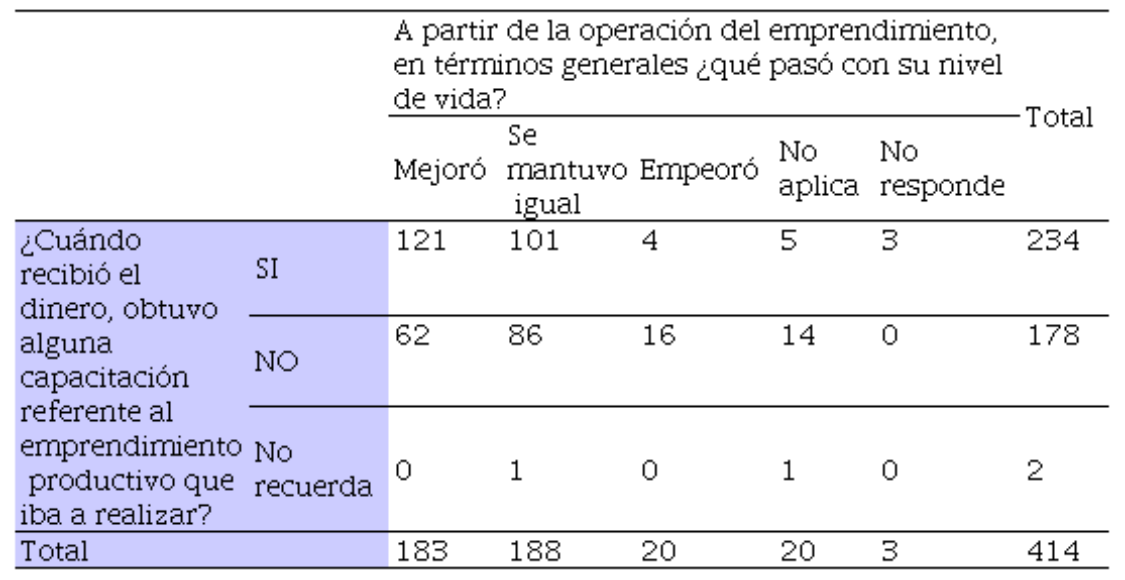

Elaborado por los autores.

En relación al proceso de capacitación y asistencia técnica, se puede observar en la tabla 4, que, de 234 encuestados, el $51.7 \%$ señala que mejoró su nivel de vida, el $43.1 \%$ que se mantuvo igual y apenas el $5.1 \%$ señala que empeoró, no aplica o no responde. Por otro lado, el $34.8 \%$ de las personas que no recibieron dicha capacitación manifiestan que su nivel de vida ha mejorado, el $48.3 \%$ que se mantiene igual y el 16,8\% que ha empeorado o no aplica. De los 183 beneficiarios que señalan mejora en su nivel de vida, el 66.1\% de ellos recibieron capacitación y si se analiza el otro extremo, es decir, las personas que expresan que su nivel de vida empeoró, el $80 \%$ no recibió capacitación, ratificando que una variable de influencia para mejorar el efecto 
económico y social del crédito para emprendimientos productivos es la formación y el acompañamiento (Rivera, 2014; Guamán et al., 2019)

Lo señalado muestra que la capacitación brindada, sin llegar a ser determinante, evidencia una influencia significativa en la mejora o no del nivel de vida.

TABLA 5.

Tabla cruzada ¿Una vez que recibió el crédito de la Institución, usted fue visitado por algún funcionario con el objeto de realizar un seguimiento a su emprendimiento? *A partir de la operación del emprendimiento en términos generales ¿qué pasó con su nivel de vida?

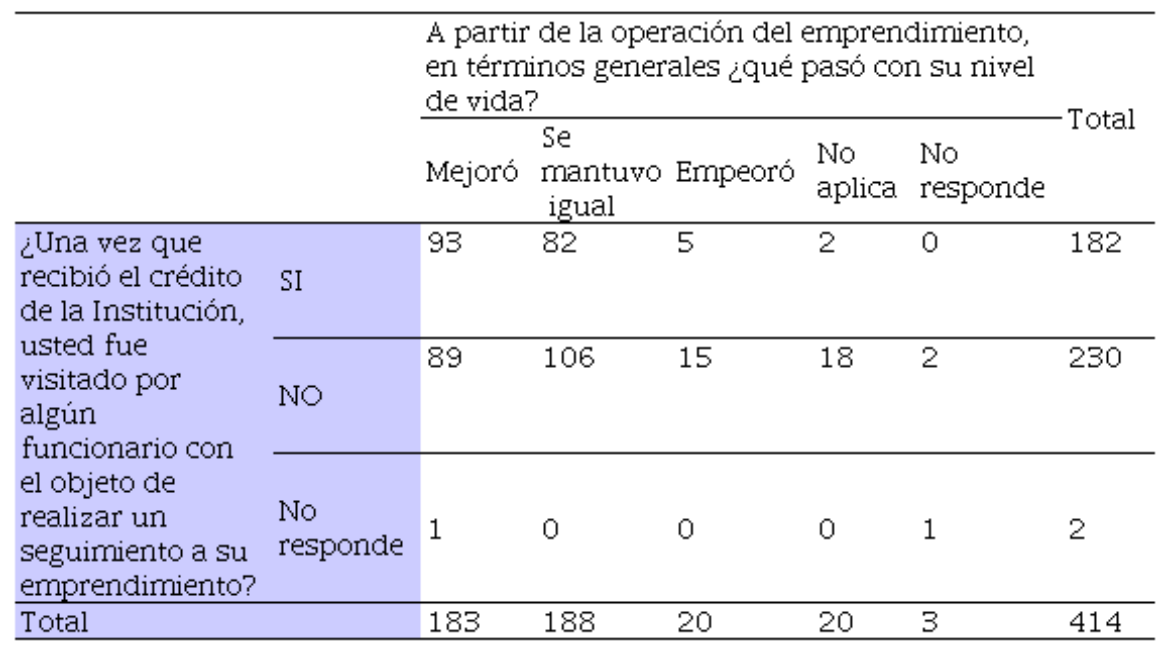

Elaborado por los autores.

$\mathrm{Al}$ analizar la tabla 5 , se puede colegir que algo más del $50 \%$ de los beneficiarios que fueron visitados, por algún funcionario del MIES, señalaron que su nivel de vida mejoró, por otro lado, el 38.6\% de los encuestados que perciben una mejora en el nivel de vida no recibieron ningún seguimiento por parte de algún técnico del Ministerio. A su vez y tomando como referencia a los beneficiarios que no recibieron ninguna visita técnica, el 38.7\% señala que mejoró su nivel de vida y el 52.6 que se mantuvo o empeoró, respaldando los informes presentados por ONU (2015) y PNUD (2016).

Lo señalado muestra que existe un limitado seguimiento por parte del MIES a los créditos financiados, el 55.6\% de los encuestados nunca fueron visitados. Si bien es cierto, el porcentaje de percepción de mejora de nivel de vida es mayor en los emprendimientos visitados, su diferencia no es significativa con aquellos en los que no hubo seguimiento. Por lo señalado, la entidad ejecutora del crédito no ha visitado a un porcentaje considerable de emprendedores y a su vez, los resultados de las visitas técnicas no han generado logros plausibles, dado que las percepciones entre los beneficiarios visitados o no, no marcan una diferencia considerable. 
TABLA 6.

Tabla cruzada. Cuando recibió el dinero ¿Obtuvo alguna capacitación referente al emprendimiento productivo que iba a realizar? ${ }^{*}$ Percepción de los emprendimientos realizados por los beneficiarios de los créditos

\begin{tabular}{|c|c|c|c|c|c|c|}
\hline & & \multicolumn{4}{|c|}{$\begin{array}{l}\text { Percepción de los emprendimientos } \\
\text { realizados por los beneficiarios de los } \\
\text { créditos }\end{array}$} & \multirow[t]{2}{*}{ Total } \\
\hline & & Éxito & Fracaso & $\begin{array}{l}\text { No } \\
\text { aplica }\end{array}$ & $\begin{array}{l}\text { No } \\
\text { responde }\end{array}$ & \\
\hline \multirow{4}{*}{$\begin{array}{l}\text { ¿Cuándo recibió el } \\
\text { dinero, obtuvo } \\
\text { alguna capacitación } \\
\text { referente al } \\
\text { emprendimiento } \\
\text { productivo que iba a } \\
\text { realizar? } \\
\text { Total }\end{array}$} & SI & 172 & 60 & 0 & 2 & 234 \\
\hline & NO & 112 & 60 & 5 & 1 & 178 \\
\hline & No recuerda & 1 & 0 & 1 & 0 & 2 \\
\hline & & 285 & 120 & 6 & 3 & 414 \\
\hline
\end{tabular}

Elaborado por los autores.

La tabla 6 refleja que el 73,5\% de los emprendimientos que tuvieron éxito recibieron alguna capacitación sobre la inversión a realizar, mientras que el 25.6\%, aunque no tuvo capacitación también tuvo éxito, sin embargo, al analizar aquellas iniciativas productivas que no recibieron capacitación al rededor del $62.9 \%$ tuvo éxito y el 33.9\% terminó en fracaso. Si bien el porcentaje de éxitos es mayor en aquellos emprendimientos que recibieron capacitación, la diferencia no es sustancialmente significativa, apenas es el 10\%. Las razones pueden ser varias, para explicar cómo un emprendimiento puede llegar a tener éxito si se tiene claramente determinado en qué actividad se va invertir y/o si se tiene experiencia sobre la ejecución de la misma y no específicamente en el hecho de recibir capacitación o no (Rivera, 2014), siendo importante para emprender la incorporación de conocimiento especializado, acompañamiento técnico y capacitación (Salinas y Osorio, 2012)

Lo señalado también se corrobora al observar que de los 120 emprendimientos que se perciben como fracasos, el 50\% tuvieron capacitación y la otra mitad no la tuvieron, entendiendo que el éxito de estas iniciativas puede ser multifactorial, las razones más importantes fueron por la muerte de los animales, en segundo lugar los que manifestaron que los animales se enferman, en tercer lugar por lo insuficiente del monto del crédito y con reducidos porcentajes están una serie de respuestas con múltiples razones para el fracaso del proyecto, más se reitera que tal como se ha impartido la capacitación, no se ha constituido en un factor diferenciador para el éxito de los emprendimientos (Salinas y Osorio, 2012).

TABLA 7.

¿En la actualidad continúa el emprendimiento?

\begin{tabular}{llllll}
\hline & & Frecuencia & Porcentaje & $\begin{array}{l}\text { Porcentaje } \\
\text { válido }\end{array}$ & $\begin{array}{l}\text { Porcentaje } \\
\text { acumulado }\end{array}$ \\
\hline \multirow{4}{*}{ Válido } & SI & 275 & 66,4 & 66,4 & 66,4 \\
\cline { 2 - 6 } & NO & 120 & 29,0 & 29,0 & 95,4 \\
\cline { 2 - 6 } & No aplica & 15 & 3,6 & 3,6 & 99,0 \\
\cline { 2 - 6 } & $\begin{array}{l}\text { No } \\
\text { responde }\end{array}$ & 4 & 1,0 & 1,0 & 100,0 \\
\cline { 2 - 6 } & Total & 414 & 100,0 & 100,0 & \\
\hline
\end{tabular}

Elaborado por los autores. 
$\mathrm{Al}$ analizar la Tabla 7 y descontando los 19 créditos que nunca se invirtieron en los emprendimientos, ya sea porque se gastaron el dinero recibido en otras actividades, como mejorar las viviendas, dar educación a sus hijos, o porque simplemente no quisieron proporcionar la información, se puede colegir que de las 395 iniciativas financiadas, alrededor de la tercera parte se liquidaron, reflejando pérdida de recursos para el Estado y la no mejoría de las condiciones de vida para este grupo destinatario; si bien, el 69,6\% de los emprendimientos se mantienen, a futuro sería importante determinar en qué condiciones y cuán sostenibles son (Rivera, 2014; PNUD, 2016).

TABLA 8.

A partir de la operación del emprendimiento en términos generales ¿qué pasó con su nivel de vida?

\begin{tabular}{llllll}
\hline \multirow{5}{*}{ Válido } & Frecuencia & Porcentaje & $\begin{array}{l}\text { Porcentaje } \\
\text { válido }\end{array}$ & $\begin{array}{l}\text { Porcentaje } \\
\text { acumulado }\end{array}$ \\
\hline \multirow{4}{*}{$\begin{array}{l}\text { Mejoró } \\
\text { Se mantuvo } \\
\text { igual }\end{array}$} & 183 & 48,2 & 44,2 & 44,2 \\
\cline { 2 - 6 } & 188 & 45,4 & 45,4 & 89,6 \\
\cline { 2 - 6 } & Empeoró & 20 & 4,8 & 4,8 & 94,4 \\
\cline { 2 - 6 } & No aplica & 19 & 4,8 & 4,8 & 99,3 \\
\cline { 2 - 6 } & No responde & 4 &, 7 &, 7 & 100,0 \\
\hline
\end{tabular}

Elaborado por los autores

Finalmente, al relacionar el nivel de vida de los beneficiarios en función de la implementación del emprendimiento (ver Tabla 8) y descartando aquellas personas que no contestaron o que no aplica la encuesta, se muestran datos preocupantes como que el $48.1 \%$ de los encuestados manifiestan que su nivel de vida se mantiene, por lo que el crédito productivo no ha generado el impacto deseado y que se traduce en mejorar las condiciones de vida, más aún el 5.12\% manifiesta que su nivel de vida empeoró, siendo importante desarrollar procesos de investigación de tipo transversal para evidenciar los cambios generados con los créditos para emprendimientos productivos en distintos momentos del tiempo, debiendo seguir procesos de análisis más complejos para un entendimiento más profundo del efecto de las variables estudiadas, siendo las recomendaciones de Aldás y Uriel (2017).

Del $46.8 \%$ de personas que señalan que su nivel de vida mejoró, el $7.2 \%$ señala que fue en salud, el $4.3 \%$ en educación, el mismo porcentaje en alimentación, el $1.2 \%$ respondió en vivienda y el $0.2 \%$ en vestimenta; el mayor porcentaje, $82.8 \%$ corresponde a varios indicadores combinados entre salud, vivienda, educación, evidenciando la necesidad de realizar una revisión profunda de las políticas y los mecanismos en las que se entrega dicho financiamiento, tomando en consideración las recomendaciones emitidas por los encuestados, las cuales se sintetizan en: $19.6 \%$ que se incremente el monto del crédito, el $12.6 \%$ que debe haber una capacitación continua, un 10.6\% manifiestan que no debe hacerse ningún cambio (corresponden a los que si recibieron capacitación y están conformes con el monto del crédito recibido), el $7 \%$ señalan que a pesar de las limitaciones del programa actual debería mantenerse; con porcentajes más pequeños están una serie de sugerencias, relacionadas a mejorar el seguimiento y que se definan mejoras para la obtención del crédito, entre otras, respaldando los hallazgos de Medina (2016) y las recomendaciones de los informes presentados por ONU (2015) y PNUD (2016).

\section{Conclusiones}

Es necesario precisar que los resultados de la presente investigación fueron obtenidos de la población que habita en la zona rural de la provincia de Pichincha, por lo cual cualquier extrapolación que se desee realizar a 
otra zona geográfica debe tener muy en cuenta, no sólo las similitudes, sino fundamentalmente las diferencias que existen entre las diversas regiones.

La investigación fue dirigida a evaluar el impacto del Plan de Crédito para emprendimientos productivos otorgado por el Gobierno de Rafael Correa por intermedio del MIES a quienes son beneficiarios del Bono de Desarrollo Humano, objetivo que se evidencia en el estudio de campo respecto a las variables de investigación consideradas para medir el grado de percepción de la población beneficiaria de dicho bono, respecto a las mejoras en sus emprendimientos y situación socio económica (Gasparini et al., 2014); situación que debe ser analizada por los organismos competentes para potenciar el crédito en beneficio del desarrollo de este sector, considerando mejorías en la forma de concesión y en el seguimiento del destino de estos recursos, en respaldo de lo planteado por Guamán, et al. (2019).

El número de encuestas aplicadas supera al tamaño óptimo de la muestra para universos finitos, alcanzando un total de 414 encuestas, esto junto con el indicador de fiabilidad Alfa de Cronbach (71,7\%), garantiza la fiabilidad de la investigación. Sin embargo, se debe reconocer como limitación que el instrumento mide únicamente el grado de percepción de los beneficiarios del crédito y no contrasta su situación social antes y después, debido a la falta de información de partida.

Sobre el trámite que tuvieron que realizar los beneficiarios para obtener el préstamo, la mayoría no tuvo ningún problema ya que al $67.1 \%$ le pareció fácil, lo que preocupa profundamente es que el $43 \%$ de beneficiarios del crédito no recibieron capacitación para optimizar el empleo de los recursos entregados, pudiendo ser una de las razones para el fracaso de algunos emprendimientos, corroborando lo mencionado por Patanakul, et al. (2016) sobre la necesidad de implementar programas paralelos de capacitación para la administración financiera en los hogares de los beneficiarios. El estudio de Barreno et al. (2018) muestra la existencia de gran variedad de emprendimientos productivos en la zona rural objeto de estudio, sin embargo, por ser la investigación en el área rural la mayoría de ellos están dentro del sector agropecuario, predominando el pecuario al agrícola, según se pudo constatar en los hallazgos de la investigación.

Otro elemento motivo de preocupación es la falta de seguimiento a los emprendimientos productivos por parte de la entidad ejecutora, siendo también causa para el fracaso y la quiebra del emprendimiento, ya que al 55.67\% de los emprendedores no los visitaron los gestores del programa. Pese a los problemas señalados, es preciso señalar que el $68.8 \%$ de las personas que recibieron el crédito para emprendimientos productivos y que lograron emprender, tuvieron éxito, sin embargo no hay que dejar de lado al $29 \%$ que reconocieron que su proyecto fue un fracaso, a las razones falta de capacitación y seguimiento entre las más importantes se suman otras muy diversas, las que tendrán que ser tomadas en cuenta para la asignación del crédito para nuevos emprendimientos que hasta la fecha se sigue entregando, lo cual, respalda los resultados evidenciados por Medina (2016) sobre los efectos del BDH en la sociedad, así como los datos presentados por PNUD (2014), PNUD (2016) y la ONU (2015).

Un aspecto igual de importante, que es necesario señalar, corresponde al total de emprendimientos que hasta el día de la toma de datos siguen activos corresponde al 66.4\%, si bien esto es satisfactorio porque constituye parte del éxito de las familias beneficiarias, no se debe dejar a un lado el 29\% que ya no tiene el emprendimiento, por las razones ya señaladas. Ante esta realidad, las propuestas de cambio expresadas en las recomendaciones, apuntan fundamentalmente a que el programa entregue mayor cantidad de dinero a cada emprendedor y que exista un programa de capacitación y seguimiento continuos.

El resultado de la investigación que permitió confirmar la hipótesis de estudio y analizar con claridad el efecto directo de la aplicación de este importante programa aplicado para las personas de escasos recursos, muestra que la ejecución y la operación de los pequeños emprendimientos productivos financiados con créditos otorgados a los beneficiarios del $\mathrm{BDH}$, han mejorado de manera significativa su percepción respecto a su nivel de vida pues, el 44,2\% señala haber mejorado su nivel de vida, el 45,4\% se mantuvo su nivel de vida inicial, tan solo un mínimo porcentaje $5.1 \%$ desmejoró. Estos datos consideran el acceso a alimentación, 
educación, salud y vivienda y un alto porcentaje $82,8 \%$ a una serie de combinaciones que involucra a todos ellos, respaldando los estudios de Gasparini et al. (2014) y Cabezas (2018).

Lo señalado se sintetiza en función a la percepción de los destinatarios del $\mathrm{BDH}$, que han recibido del Estado por medio de los créditos productivos, recursos económicos que buscan atender necesidades prioritarias de estos segmentos e identificar si dichos recursos fueron utilizados de manera productiva o fortalecieron sus emprendimientos, respaldando otras investigaciones como las realizadas por Ponce, et al. (2013), en el cual se señala que el BDH no ha generado impacto plausible en educación, evaluado a partir de elevar el nivel cognitivo de los niños/as de esos hogares, o al menos no existe evidencia empírica que demuestre lo contrario; así como, el estudio de Calvas (2010) que demuestra que el BDH no presentó ninguna evidencia estadísticamente significativa de mejora en la inserción educativa.

Finalmente, en base a los resultados de la investigación se concluye que en la zona investigada, el impacto que han tenido los créditos para emprendimientos productivos por las personas que reciben el bono de desarrollo humano, si bien, han permitido mejorar el nivel de vida del $44.2 \%$ de los beneficiarios, el $46.8 \%$ restante no lo ha logrado, por lo tanto se considera que los recursos entregados por parte del Gobierno para tal efecto no han sido optimizados al cien por ciento y que en el futuro se deberá considerar todos los elementos que se han señalado como problemas para que sean corregidos.

$\mathrm{Al}$ ser un estudio de caso que toma como referencia geográfica la población rural de la Provincia de Pichincha en Ecuador, se han presentado varias limitaciones que deben ser consideras por autores que deseen profundizar o ampliar en el tema; siendo en primer lugar la dificultad de acceso a la información de campo al encontrarse hogares beneficiarios del $\mathrm{BDH}$ y de créditos productivos en zonas sin acceso a transporte y de caminos irregulares impidiendo poder retornar por información al no encontrar, en una primera instancia, a los sujetos de estudio; en segundo lugar se reconoce como limitación la falta de actualización de los datos personales de beneficiarios respecto a direcciones, teléfonos de contacto o personas de referencia, mostrando un importante número de datos errados; en tercer lugar, para futuros estudios se debe considerar diferencias culturales, de niveles de educación, así como otras características de la población que pueden cambiar en las distintas zonas y provincias del Ecuador, al reconocerse como un país multicultural y pluriétnico.

\section{REFERENCIAS BIBLIOGRÁFICAS}

Acosta, A., y Falconí, F. (2005). Asedios a lo imposible: propuestas económicas en construcción. (FLACSO, Ed.). Quito. Aldás, J., y Uriel, E. (2017). Análisis multivariante aplicado con R (Segunda). Madrid, España.: Ediciones Paraninfo.

Armas, A. (2005). Redes e institucionalización en Ecuador. Bono de Desarrollo Humano. Retrieved from http://reposi torio.cepal.org/bitstream/handle/11362/5796/S05828_es.pdf?sequence $=1$

Artaraz, M. (2002). Teoría de las tres dimensiones de desarrollo sostenible. Ecosistemas, 2. Retrieved from https://w ww.revistaecosistemas.net/index.php/ecosistemas/article/view/614

Barreno, L., López, H., López, M., y Gonzales, G. (2018). La precarización salarial en la actividad agrícola y su incidencia en la política de economía popular y solidaria en el Ecuador, XLI, 47-67.

Cabezas, G. (2018). Una explicación sobre los cambios distributivos para la economía ecuatoriana entre 2006 y 2014. ¿hay evidencia de crecimiento pro pobre? Cuestiones Económicas, 28.

Calderón, Á. (2012). El bono de desarrollo humano. Retrieved from http://www.eltelegrafo.com.ec/noticias/cartasal-director/1/el-bono-de-desarrollo-humano-2

Calvas, G. V. (2010a). Evaluación de impacto del bono de desarrollo bumano en la educación. Flacso. Retrieved from h ttps://repositorio.flacsoandes.edu.ec/handle/10469/2405

Calvas, G. V. (2010b). Evaluación de Impacto del Bono de Desarrollo Humano en la Educación, 97.

CEPAL. (2006). La protección social de cara al futuro: acceso, financiamiento y solidaridad. Naciones Unidas, Capítulo I. Retrieved from http://www.cepal.org/ilpes/noticias/paginas/5/39245/Proteccion_social_de_cara_al_futur o.pdf 
Coba, E., y Díaz, J. (2014). Analíti a. Analítika.

Consejo Nacional de Planificación. (2017). Plan Nacional Para el Buen Vivir 2017-2021. Retrieved from http://www.planificacion.gob.ec/wp-content/uploads/downloads/2017/07/Plan-Nacional-para-el-Bue n-Vivir-2017-2021.pdf

Cortés, J., y Cisneros, G. (2014). Microempresas: Indicadores económicos macro, ayudas gubernamentales, y análisis del sector microempresarial de Cotocollao. Revista Retos, 4, 73-88.

Gasparini, L., Cicowiez, M., y Sosa, W. (2014). Pobreza y Desigualdad en America Latina: Conceptos, Herramientas y Aplicaciones Leonardo. Documentos de Trabajo Del CEDLAS. Retrieved from http://sedici.unlp.edu.ar/bitst $\mathrm{ream} /$ handle $/ 10915 / 50845 /$ Documento_completo__.pdf?sequence $=1 \&$ isAllowed $=\mathrm{y}$

González, J., y Pazmino, M. (2015). Cálculo e interpretación del Alfa de Cronbach para el caso de validación de la consistencia interna de un cuestionario , con dos posibles escalas tipo Likert. Revista Publicando, (February), 6277.

Guamán, J., Lara, E., Alvarado, R., y Ponce, P. (2019). Efecto del bono de desarrollo humano en el gasto en salud y educación en Ecuador utilizando propensity score matching. Economia Y Poitica, XV. https://doi.org/https:// doi.org/10.25097/rep.n30.2019.02

INEC. (2018). Reporte de pobreza y desigualdad. Quito. Retrieved from http://www.ecuadorencifras.gob.ec/docume ntos/web-inec/POBREZA/2018/Junio-2018/Informe_pobreza_y_desigualdad-junio_2018.pdf

Martínez, D., Borja, T., Medellín, N., y Cueva, P. (2017). ¿Cómo funciona el Bono de Desarrollo Humano\#?

Medina, H. (2016). "EL CRÉDITO DE DESARROLLO HUMANO\#: IMPACTOS EN LAS HELEN SUGEY MEDINA CEVALLOS TUTOR\#: ECON . MARIA ROSA ANCHUNDIA GUAYAQUIL - ECUADOR ABRIL 2016." Universidad de Guayaquil.

Ministerio de Industrias y Productividad. (2017). Emprendimientos productivos con créditos de Desarrollo Humano. Retrieved from http://www.industrias.gob.ec/gobierno-incentiva-emprendimientos-productivos-con-creditosde-desarrollo-humano/

ONU. (2015). Programa De Las Naciones Unidas Para El Desarrollo, 4. Retrieved from http://www.unaids.org/sit es/default/files/media_asset/PNUD_es.pdf

Paladines, E. (2016). EL PARADIGMA DE LA MAXIMIZACIÓN DEL BIENESTAR SOCIAL EN LA TEORÍA UTILITARISTA MODERNA DE TRIBUTACIÓN ÓPTIMA: UNA BREVE HISTORIA INTERNA DESDE UNA PERSPECTIVA ÉTICA. Cuestiones Económicas, 26. Retrieved from https://www.bce.fin.ec/cu estiones_economicas/images/PDFS/2016/No1/Documento2.pdf

Patanakul, P., Kwak, Y. H., Zwikael, O., y Liu, M. (2016). What impacts the performance of large-scale government projects? International Journal of Project Management, 34(3), 452-466. https://doi.org/10.1016/j.ijproman.2 015.12 .001

Pérez, L., \& Cardoso, R. (2014). Construcción del Buen Vivir o Sumak Kawsay en Ecuador: una alternativa al paradigma de desarrollo occidental. Contribuciones Desde Coatepec, (26), 49-66.

PNUD. (2016). Desarrollo humano para todos, 40. Retrieved from http://hdr.undp.org/sites/default/files/HDR20 16_SP_Overview_Web.pdf

PNUD, P. de N. U. para el D. (2014). Informe sobre Desarrollo Humano 2014 Equipo del Informe sobre Desarrollo Director y autor principal, 19.

Ponce, J., Enríquez, F., Molyneux, M., y Thomson, M. (2013). Hacia una reforma del Bono de Desarrollo Humano\#: Algunas reflexiones (Abya-Yala). Quito: CARE. Retrieved from https://biblio.flacsoandes.edu.ec/libros/digital 157099.pdf

Rivera, E. (2014). IMPACTO ECONÓMICO Y SOCIAL DEL BONO DE DESARROLLO HUMANO EN LA POBLACIÓN BENEFICIARIA DE LA PROVINCIA DE COTOPAXI, PERIODO 2008-2012. Universidad de las Fuerzas Armadas.

Rosero, L., y Ramos, M. (2016). El Estado del Buen Vivir - Bono de Desarrollo Humano Ecuador. Quito: Ministerio de Inclusión Económica y Social. 
Salinas, F., y Osorio, L. (2012). Emprendimiento y Economía Social , oportunidades y efectos en una sociedad en transformación. CIRIEC-España, Revista de Economia Pública, Social Y Cooperativa. Retrieved from https://w ww.redalyc.org/pdf/174/17425798008.pdf

Sarmiento, S. (2017). Evolución de la desigualdad de ingresos en Ecuador, período 2007-2015. Analítika\#: Revista de Análisis Estadistico, 13(13), 49-79.

SIISE. (2010). Crédito de Desarrollo Humano - CDH_Ficha_Metodológica. Retrieved from file://C:/Users/ hector.lopez/AppData/Local/Mendeley Ltd./Mendeley Desktop/Downloaded/SIISE - Unknown - Crédito de Desarrollo Humano - CDH_Ficha_Metodológica.htm

SIISE. (2013). Ficha metodológica. Retrieved from http://www.siise.gob.ec/siiseweb/PageWebs/Accion Social/ ficacc_OS013.htm

Stefanoni, P. (2012). Posneoliberalismo cuesta arriba. Nueva Sociedad, 3552. Retrieved from https://nuso.org/medi a/articles/downloads/3846_1.pdf

Tibocha, A. M., y Jaramillo Jassir, M. (2008). La revolución democrática de Rafael Correa. Analisis Politico, 21(64), 22-39.

Villatoro, P., y Silva, A. (2005). Políticas Sociales. Retrieved from http://repositorio.cepal.org/bitstream/handle/11 362/6085/S047600_es.pdf

Vos, R. (2000). Ecuador\#: Crisis Y Protección Social. Siise. Retrieved from http://www.siise.gob.ec/siiseweb/PageW ebs/RES/pubsii/pubsii_0044.pdf

\section{Notas}

[1] El gobierno de la revolución ciudadana se instaura desde el 2007 en el Ecuador como representación del partido político Alianza País liderado por el Eco. Rafael Correa Delgado (Tibocha y Jaramillo Jassir, 2008)

[2] Pérez y Cardoso, (2014) señalan que "El Buen Vivir" es un principio plasmado en la Constitución Ecuatoriana que se basa en el concepto ancestral del "Sumak Kawsay", en el que se hace posible no solo la permanencia de la diversidad cultural y ambiental, sino también la felicidad individual y colectiva.

[4] Proyecto integral para el fomento productivo y competitivo para las micro, pequeñas y medianas empresas que tiene como objeto contribuir a mejorar las condiciones y capacidades de las micro, pequeñas, medianas empresas y artesanos del Ecuador (Cortés y Cisneros, 2014)

[5] Programa de fomento para la Exportación de la Mipymes ecuatorianas dirigida a las micro y pequeñas empresas, para presentar proyectos que requieran de cofinanciamiento para el fomento de la oferta exportable y la internacionalización de sus productos. Este programa tiene como objeto incrementar y mejorar la productividad y competitividad, el acceso a mercados, la calidad de la producción, el fomento de la oferta exportable ecuatoriana, el desarrollo y la diversificación de productos, y servicios con valor agregado de micro y pequeñas empresas (Cortés y Cisneros, 2014

La Universidad de Cuenca en Ecuador, conserva los derechos patrimoniales (copyright) de las obras publicadas, y favorece y permite la reutilización de las mismas bajo la licencia Creative Commons AtribuciónNoComercial-CompartirIgual 4.0 Internacional (CC BY-NC-SA 4.0), por lo cual se pueden copiar, usar, difundir, transmitir y exponer públicamente, siempre que: a. Se cite la autoría y fuente original de su publicación (revista, editorial, URL y DOI de la obra). b. No se usen para fines comerciales u onerosos. c. Se mencione la existencia y especificaciones de esta licencia de uso.

CC BY-NC-SA 